\title{
Reactor antineutrinos and nuclear physics
}

\author{
A.B. Balantekin ${ }^{\mathrm{a}}$ \\ Department of Physics, University of Wisconsin, Madison, WI 53706, USA
}

Received: 8 September 2016 / Revised: 31 October 2016

Published online: 24 November 2016

(c) The Author(s) 2016. This article is published with open access at Springerlink.com

Communicated by N. Alamanos

\begin{abstract}
Short-baseline reactor neutrino experiments successfully measured the neutrino parameters they set out to measure, but they also identified a shape distortion in the $5-7 \mathrm{MeV}$ range as well as a reduction from the predicted value of the flux. Nuclear physics input into the calculations of reactor antineutrino spectra needs to be better refined if this anomaly is to be interpreted as due to sterile neutrino states.
\end{abstract}

The need for better nuclear data usually comes up in the technological and medical applications of nuclear physics. Some recent developments, however, illustrate such a need in connection with fundamental science, namely exploring new physics beyond the Standard Model. The issue is the spectra of antineutrinos coming from commercial power reactors. Even though total antineutrino output is correlated with the thermal power of the reactor, which of course is typically well monitored, the uncertainty on the energy spectra is more than desired. $99.9 \%$ of the power in these reactors comes from the fissions of only four isotopes: ${ }^{235} \mathrm{U},{ }^{238} \mathrm{U},{ }^{239} \mathrm{Pu},{ }^{241} \mathrm{Pu}$. Number of fissions per isotope can be monitored using the knowledge of the total power and simulation of the fuel composition. Revisiting the way systematic errors in the Coulomb and weak magnetism corrections are implemented and taking the charges of the decaying nuclei into account more carefully, a French group recently provided updated spectra for these decays [1]. A subsequent calculation showed that the deviation of the ratio of the observed event rate at reactors to the predicted rate is less than unity at $98.6 \%$ confidence level, a situation that was dubbed "reactor antineutrino anomaly" [2].

In parallel to these developments three experiments, Daya Bay [3], Double Chooz [4], and RENO [5], were taking data to measure the neutrino mixing angle between first and third generations, $\theta_{13}$. These experiments performed very successfully to achieve their stated goals: as a result of their measurements this mixing angle is now the most precisely known neutrino mixing angle. To observe the oscillation of antineutrinos due to $\theta_{13}$ these experiments did not need to know the predicted antineutrino energy spectrum: they measured the flux near the reactor with near detectors and compared this measurement with the flux measured at the detectors placed farther away

\footnotetext{
a e-mail: baha@physics.wisc.edu
}

at the optimum distance for oscillation due to a nonzero value of $\theta_{13}$. However, as they acquired more data at the near detectors, an unexpected feature has emerged: an excess of antineutrinos with energies around $\sim 5-7 \mathrm{MeV}$, referred to as a "bump" or "shoulder". This feature turned out to be very prominent in all three experiments, Daya Bay [6,7], Double Chooz [8], and RENO [9]. Furthermore the total antineutrino flux they measured was consistent with a reduction similar to that suggested by the reactor antineutrino anomaly.

If we assume that reactor antineutrino flux and energy spectra are indeed correctly predicted, then one has to conclude that these experiments are missing a fraction of those neutrinos. Electron antineutrinos are detected by their capture on protons. We know from solar, atmospheric and long-baseline neutrino oscillation experiments that the distance between the reactor core and even their far detectors is too short for electron antineutrinos oscillate into muon or tau antineutrinos. One may then conclude that they mix with a fourth (or more) neutrino-like state, which does not feel the standard weak interactions (or, more precisely, does not get captured on a proton). Such states are known as sterile neutrinos and they represent new physics beyond the Standard Model of particle physics. From the flux deficit and known baselines one can estimate the mixing angle and $\delta m^{2}$ (the difference between the square of their masses and the square of the mass of the active -i.e., weakly interacting state) parameter space $[10,11]$. Recently the Daya Bay Collaboration looked for and found no evidence of sterile neutrino mixing in the $2 \times 10^{-4} \mathrm{eV}^{2} \leq\left|\delta m^{2}\right| \leq 0.3 \mathrm{eV}^{2}$ mass range $[12,13]$. In fact a recent joint analysis from MINOS, Daya Bay, and Bugey-3 experiments significantly shrinks the allowed parameter space [14], but does not completely rule it out. Another recent result from the IceCube neutrino telescope at the South Pole provides limits on mixing 
between sterile states and the muon neutrinos, but does not constrain the neutrino mixing angle that would be relevant to the reactor anomaly [15].

Interpreting the reactor anomaly in terms of sterile neutrinos is a rather drastic conclusion, consequently it is certainly worthwhile to design dedicated experiments to probe neutrino oscillations over meter-long baselines [16]. The physics program of such an experiment is outlined in ref. [17]. In the meantime, however, one should explore how well the nuclear physics underlying nuclear power reactors are known, and in particular if there are more conventional explanations of the $\sim 5-7 \mathrm{MeV}$ excess. Two different approaches are employed to determine the reactor antineutrino spectra. One approach is to start with the measured electron spectra and convert it into electron antineutrino spectra. An example of this approach is given in ref. [18] where the antineutrino spectrum from ${ }^{235} \mathrm{U}$ thermal neutron fission products up to $9.5 \mathrm{MeV}$ were measured. In such an approach many fission products are measured together in a single experiment. A second approach is to directly sum the contribution of each fission fragment to the reactor antineutrino spectra using fission yields and beta-decay data from nuclear data compilations. A recent study found that the summation method indeed gives an excess of antineutrinos in the $5-7 \mathrm{MeV}$ region [19], a feature not present in the spectra obtained by the conversion method. However, another analysis using modern nuclear data libraries, raised concerns about the validity of the database used in this calculation as adjusting some of the fission rates eliminates the bump [20].

Neither the summation method nor the electron spectrum conversion method are free of systematic errors. The fission yields used in the summation method have large uncertainties. Branching ratios are not measured for some of the fragments and, as we discuss below, beta-decay spectra and rates are not always known. In the conversion method, the antineutrino flux resulting from the conversion also depends on the nuclear physics of the beta decays. The likely most fruitful approach to understanding the sensitivity of the predicted neutrino spectra to nuclear effects is to use a combination of both methods. Indeed ref. [1] used such an approach. More recently the authors of ref. [21] also utilized a hybrid approach to fit the electron spectra in good agreement with data, but they find that different treatments of the nuclear physics of the beta-decay rates led to antineutrino spectra that differ at about $4 \%$ level.

One of the key ingredients of the summation method is the nuclear beta decay rates. Subtleties in the calculations of those rates may not be immediately obvious to the casual observer. Some aspects are well understood, but not necessarily easy to implement. Since nuclei and nucleons are not point particles one has to introduce form factors in writing the vector and axial-vector nuclear currents. In some of the earlier compilations of beta-decay rates the vector form factor that depends on the spin tensor (called weak magnetism) was taken into account using a linear approximation. The Coulomb corrections (due to the electromagnetic final-state interaction between the daughter nucleus and the outgoing charged lepton) were similarly simplified. In fact, a reexamination of these ap- proximations in refs. [1] and [2] led to the identification of the reactor neutrino anomaly. Indeed reactor neutrino models $[1,22]$ used by the three short-baseline experiments include a critical evaluation of the errors in those approximations. However, a careful analysis of the underlying nuclear physics suggests that reactor antineutrino fluxes are not known to the accuracy suggested by the current models [23].

In calculating nuclear beta decays, the wave functions for the leptons are taken to be plane waves, which in itself is an excellent approximation. However, in a further approximation, noting that lepton energies are in the order of several $\mathrm{MeV}$, the term $\mathbf{k} \cdot \mathbf{r}$ in the exponent is assumed to be very small, and the wave functions are replaced by the first term in the expansion, i.e., suitably, normalized constants. This is referred to as the "allowed" approximation. Sometimes $\mathbf{k} \cdot \mathbf{r}$ cannot be ignored, but the expansion of the exponential is truncated after a few more terms. These are called "first-forbidden", "second-forbidden", etc. approximations depending on whether the expansion is terminated after the the term with first, second, etc. power of $\mathbf{k} \cdot \mathbf{r}$. Note that, because of the position vector, forbidden approximations to the beta decay introduce the orbital angular momentum, potentially much complicating the calculations. A careful analysis concludes that the corrections that lead to the reactor antineutrino anomaly are uncertain for the $30 \%$ of the flux that arises from forbidden decays [21]. Another uncertain aspect of the beta-decay rate relates to the axial-vector component of the weak current. Since the vector current is conserved, it remains the same as one moves from the pointlike constituents to nucleons and nuclei. In contrast, the axial vector is only partially conserved. Its strength, $g_{A}$, needs to be quenched in calculations of nuclear matrix elements in weak interactions. The reasons for and the amount of this quenching are somewhat obscure. Some of it comes from the matter effects, and some from the necessary truncation of the model spaces. The exact value of $g_{A}$ could be crucial in many contexts; for example half-lives for the neutrinoless double-beta decay depend on the fourth power of $g_{A}$. Another correction to the beta-decay rates comes from the finite size of the nucleus [24]. In the light of these theoretical difficulties, the experimental input would be crucial to understand the reactor anomaly. A recent measurement provides a good example: three beta decays ${ }^{92} \mathrm{Rb},{ }^{96} \mathrm{Y}$, and ${ }^{142} \mathrm{Cs}$ contribute $43 \%$ of the antineutrino flux emitted by nuclear reactors near $5.5 \mathrm{MeV}$. The latest measurement of these beta decays substantially modifies the feedings of ${ }^{142} \mathrm{Ba}$ from ${ }^{142} \mathrm{Cs}$ decays, increasing the discrepancy between the observed and the expected reactor antineutrino flux between 5 and $7 \mathrm{MeV}$ [25].

A second key ingredient of the summation method is the fission yields as emphasized in ref. [20]. The shape of the shoulder in the antineutrino energy spectra is sensitive to these yields [23]. It was argued that ${ }^{235} \mathrm{U}$ fission fragments are the main contributors to the neutrino anomaly [26], this assertion is likely to be correct, but it needs to be experimentally tested. Since a good number of such fragments are radioactive, facilities with exotic nuclear beams could help [27]. As one uses fission yields as 
an input into the calculations of the reactor antineutrino spectra, subtle issues may also arise. For example, decay rates for the vast majority of antineutrino producing nuclides are directly related to the fission rate. But some of the fission products may capture neutrons before they beta decay. This introduces a nonlinear dependence on the neutron flux and a nonlinear correction to the neutrino flux, which should be included in the estimates [28].

For a more technical discussion of the salient nuclear physics issues associated with the reactor antineutrino anomaly the reader is referred to ref. [29]. Both the betadecay rates and the fission fragment distributions used as an input in the calculations of the reactor antineutrino spectra need to be better refined if this anomaly is to be interpreted as due to sterile neutrino states.

I thank B. Holstein for a useful discussion. This work was supported in part by the US National Science Foundation Grant No. PHY-1514695.

Open Access This is an open access article distributed under the terms of the Creative Commons Attribution License (http://creativecommons.org/licenses/by/4.0), which permits unrestricted use, distribution, and reproduction in any medium, provided the original work is properly cited.

\section{References}

1. T.A. Mueller et al., Phys. Rev. C 83, 054615 (2011).

2. G. Mention, M. Fechner, T. Lasserre, T.A. Mueller, D. Lhuillier, M. Cribier, A. Letourneau, Phys. Rev. D 83, 073006 (2011)

3. Daya Bay Collaboration (F.P. An et al.), Phys. Rev. Lett. 108, 171803 (2012).

4. Double Chooz Collaboration (Y. Abe et al.), Phys. Rev. Lett. 108, 131801 (2012).

5. RENO Collaboration (J.K. Ahn et al.), Phys. Rev. Lett. 108, 191802 (2012).

6. Daya Bay Collaboration (F.P. An et al.), Phys. Rev. Lett. 116, 061801 (2016).
7. Daya Bay Collaboration (F.P. An et al.), arXiv:1607.05378 [hep-ex].

8. Double Chooz Collaboration (Y. Abe et al.), JHEP 01, 163 (2016).

9. RENO Collaboration (J.H. Choi et al.), Phys. Rev. Lett. 116, 211801 (2016).

10. J. Kopp, M. Maltoni, T. Schwetz, Phys. Rev. Lett. 107, 091801 (2011).

11. C. Giunti, M. Laveder, Phys. Rev. D 85, 031301 (2012).

12. Daya Bay Collaboration (F.P. An et al.), Phys. Rev. Lett. 117, 151802 (2016).

13. Daya Bay Collaboration (F.P. An et al.), Phys. Rev. Lett. 113, 141802 (2014).

14. Daya Bay and MINOS Collaborations (P. Adamson et al.), Phys. Rev. Lett. 117, 151801 (2016).

15. IceCube Collaboration (M.G. Aartsen et al.), Phys. Rev. Lett. 117, 071801 (2016).

16. K.N. Abazajian et al., arXiv:1204.5379 [hep-ph].

17. See, e.g., PROSPECT Collaboration (J. Ashenfelter et al.), J. Phys. G 43, 113001 (2016).

18. K. Schreckenbach, G. Colvin, W. Gelletly, F. Von Feilitzsch, Phys. Lett. B 160, 325 (1985).

19. D.A. Dwyer, T.J. Langford, Phys. Rev. Lett. 114, 012502 (2015).

20. A.A. Sonzogni, E.A. McCutchan, T.D. Johnson, P. Dimitriou, Phys. Rev. Lett. 116, 132502 (2016).

21. A.C. Hayes, J.L. Friar, G.T. Garvey, G. Jungman, G. Jonkmans, Phys. Rev. Lett. 112, 202501 (2014).

22. P. Huber, Phys. Rev. C 84, 024617 (2011) 85, 029901(E) (2012).

23. A.C. Hayes, J.L. Friar, G.T. Garvey, D. Ibeling, G. Jungman, T. Kawano, R.W. Mills, Phys. Rev. D 92, 033015 (2015).

24. X.B. Wang, J.L. Friar, A.C. Hayes, Phys. Rev. C 94, 034314 (2016).

25. B.C. Rasco et al., Phys. Rev. Lett. 117, 092501 (2016).

26. C. Giunti, arXiv:1608.04096 [hep-ph].

27. A.B. Balantekin et al., Mod. Phys. Lett. A 29, 1430010 (2014).

28. P. Huber, P. Jaffke, Phys. Rev. Lett. 116, 122503 (2016).

29. A.C. Hayes, P. Vogel, arXiv:1605.02047 [hep-ph]. 